\title{
Update on Cardiovascular Benefits with Sodium Glucose Cotransporter-2 Inhibitors (SGLT-2i)
}

\author{
Nana M and Bondugulapati LNR* \\ Diabetes \& Endocrine Unit, Maelor Hospital, United Kingdom
}

*Corresponding author: LNR Bondugulapati, Consultant Physician \& Endocrinologist, Gladstone Centre, Maelor Hospital, Wrexham, UK.

Received Date: May 13, 2019

Published Date: May 16, 2019

\section{Introduction}

The prevalence of type 2 diabetes mellitus (T2DM) has reached epidemic proportions. The global prevalence of diabetes among adults over 18 years of age has risen from $4.7 \%$ in 1980 to $8.5 \%$ in 2014 [1]. Approximately $90 \%$ of these patients are expected to have T2DM based on the population-based surveys in developed countries [2]. The latest estimates by the international diabetes federation project that 592 million adults (1 in 10 persons) worldwide will have DM by 2035 [3]. Patients with T2DM are at significantly higher risk for cardiovascular disease (CVD) and as such, CVD represents the most prevalent cause of mortality and morbidity in this group [4]. In addition to the standard risk factors for CVD like obesity, dyslipidaemia and hypertension, which are common comorbidities seen in majority of T2DM patients, there are studies reporting of additional risk factors in these patients including hypercoagulability, endothelial dysfunction, chronic kidney disease, autonomic neuropathy and enhanced oxidative stress which may directly contribute to the development of CVD [5].

There has been a colossal change in the T2DM treatment options in the last decade with several new classes of drugs emerging including dipeptidyl peptidase-4 inhibitors, glucagon-like peptide 1 agonists and sodium glucose cotransporter- 2 inhibitors (SGLT-2i). Further to United States Food \& Drug Administration (US FDA) recommendations, all the new drugs now need to go through cardiovascular (CV) safety studies and prove non-inferiority. Surprisingly, SGLT-2i not only demonstrated CV safety but in fact were shown to reduce heart failure admissions and improve major adverse cardiac event (MACE) outcomes in patients with established cardiovascular disease. This mini-review is focussed on summarising the cardiovascular benefits of this exciting class of new drugs.

\section{SGLT-2 inhibitors and their Pharmacodynamics}

Three main drugs that are approved for usage in T2DM patients by the US FDA from this class are canagliflozin, dapagliflozin and empagliflozin (approved in March 2013, Jan 2014 and Aug 2014 respectively). SGLT-2i act by inhibiting the reabsorption of filtered glucose in the proximal convoluted tubule (PCT) of the kidneys, a mechanism that is independent of insulin pathway and therefore not affected by beta cell decline. Thus, they can be prescribed at any stage of T2DM. Apart from the basic function of antihyperglycemic effect, these drugs also help improve other metabolic parameters including hypertension, obesity and to some extent dyslipidaemia too. This makes them the preferred drug in patients with these comorbidities.

The entire blood volume is filtered by the kidneys about 40-50 times/day in an average healthy adult with daily filtration rate of 160-180 grams of glucose. This filtered glucose is absorbed back almost completely in the kidneys through the proximal tubules via sodium-dependent transmembrane protein family called sodium glucose cotransporters (SGLT1 and SGLT2) preventing glycosuria. While SGLT1 is a low-capacity high-affinity transporter that is responsible for reabsorption of $10 \%$ of glucose, SGLT2 is a highcapacity low-affinity transporter and reabsorbs the rest of the glucose (90\%). The SGLT-2i inhibit SGLT2 in the PCT and leading to increased urinary glucose excretion and reduction in blood glucose levels [6]. They are also shown to preserve beta cell function [7].

The common side effects include thrush, balanitis and urinary tract infections. In view of osmotic diuresis, volume depletion is a potential risk especially in patients who are on diuretics. Hypoglycaemia is rare except when these drugs are used concurrently with either insulin or insulin secretagogues. There are few case reports of diabetic ketoacidosis (DKA) especially when used in patients with type 1 diabetes mellitus (unlicensed usage). Very rarely, Fournier's gangrene has also been reported [8].

\section{Cardiovascular Outcome Trials with SGLT-2 inhibitors}

Further to adverse cardiac events post marketing with rosiglitazone, the US FDA made recommendations that glucoselowering medications used for T2DM should prove their 
cardiovascular safety by achieving a maximum hazard ratio of 1.30 (upper 95\% of confidence interval) in a cardiovascular outcome trial (CVOT) with a composite endpoint of first myocardial infarction (MI), stroke or cardiovascular death events [9]. European Medicines Agency (EMA) followed suit in 2012 and published a guideline on CVOTs for all the anti-diabetic therapies. Accordingly, three CVOT studies were reported where SGLT-2i not only showed their CV safety but also proved their CV efficacy in T2DM patients. These are summarised below.

In the "Empagliflozin Cardiovascular Outcome Event Trial in Type 2 Diabetes Mellitus Patients-Removing Excess Glucose (EMPA-REG OUTCOME)" study, which was the first randomized, double-blinded, placebo-controlled trial for this class, reported by Zinman et al., they have randomly assigned adults with T2DM who had established cardiovascular disease to receive $10 \mathrm{mg}$ or $25 \mathrm{mg}$ of empagliflozin (4687 patients) or placebo (2333 patients) once daily against a background of standard care. A total of 7020 patients were treated with a median observation time of 3.1 years. The primary composite outcome was death from CV causes, nonfatal myocardial infarction or nonfatal stroke. The key secondary composite outcome was the primary outcome plus hospitalization for unstable angina. The primary outcome occurred in $10.5 \%$ in the pooled empagliflozin group and in $12.1 \%$ in the placebo group (hazard ratio in the empagliflozin group, 0.86; 95.02\% confidence interval 0.74 to 0.99 ; $\mathrm{P}=0.04$ for superiority). In the empagliflozin group there were significantly lower rates of death from cardiovascular causes (3.7\%, vs. 5.9\%; 38\% relative risk reduction), hospitalization for heart failure $(2.7 \%$ and $4.1 \%$; $35 \%$ relative risk reduction), and death from any cause (5.7\% and 8.3\%; $32 \%$ relative risk reduction). There was no significant betweengroup difference in the key secondary outcome [10].

This study was followed by "The Canagliflozin Cardiovascular Assessment Study (CANVAS)" in which researchers integrated data from two trials (CANVAS and CANVAS-Renal) involving a total of 10,142 participants with T2DM and high cardiovascular risk. Participants in CANVAS group were randomly assigned in a 1:1:1 ratio to receive canagliflozin at a dose of $300 \mathrm{mg}$, or 100 $\mathrm{mg}$, or matching placebo. Participants in CANVAS-R were randomly assigned in a 1:1 ratio to receive canagliflozin, $100 \mathrm{mg}$ daily initially with an optional increase to $300 \mathrm{mg} /$ day starting from week 13, or matching placebo who were then followed for a mean of 188.2 weeks. The primary outcome was a composite of death from cardiovascular causes, nonfatal myocardial infarction, or nonfatal stroke. The mean age of the participants was 63.3 years with the mean duration of diabetes of 13.5 years and nearly $2 / 3$ of patients had a history of CV disease. Similarly to the outcomes observed in the EMPA-REG outcome study, the rate of the primary outcome was lower with canagliflozin than with the placebo (occurring in 26.9 vs. 31.5 participants per 1000 patient-years; hazard ratio 0.86 ; $95 \%$ confidence interval 0.75 to $0.97 ; \mathrm{P}<0.001$ for noninferiority; $\mathrm{P}=0.02$ for superiority). There was also a possible suggestion of benefit in the canagliflozin group in terms of progression of albuminuria (hazard ratio $0.73 ; 95 \% \mathrm{CI}, 0.67$ to 0.79 ) and the composite outcome of a sustained $40 \%$ reduction in the estimated glomerular filtration rate, the need for renal-replacement therapy, or death from renal causes (hazard ratio, $0.60 ; 95 \% \mathrm{CI}, 0.47$ to 0.77 ). Although adverse reactions were mostly akin to previous reports, an increased risk of amputations were also noted (6.3 vs. 3.4 participants per 1000 patient-years) which were primarily peripheral at the level of toes or metatarsals [11].

In a sub-analysis, canagliflozin reduced the overall risk of heart failure events in patients with T2DM who had high CV risk irrespective of their ejection fraction i.e. with no clear efficacy difference in patients with reduced versus preserved ejection fraction. This can potentially open new doors to diabetic patients having heart failure with preserved ejection fraction, where no other intervention has been shown to have clinically meaningful benefits thus far $[12,13]$.

In "Dapagliflozin Effect on Cardiovascular Events trial" (DECLARE-TIMI 58), investigators randomly assigned T2DM patients with atherosclerotic cardiovascular disease (ASCVD) or who were at risk for ASCVD to receive either dapagliflozin or placebo. The primary safety outcome was a composite of MACE, defined as cardiovascular death, myocardial infarction, or ischemic stroke. The primary efficacy outcomes were MACE and a composite of cardiovascular death or hospitalization for heart failure. Secondary efficacy outcomes were a renal composite and death from any cause. This is the largest CVOT trial of the class with 17,160 patients, including $59.36 \%$ patients $(10,186)$ without ASCVD, which also had longest follow-up (median 4.2 years). In the primary safety outcome analysis, dapagliflozin was noninferior to placebo with regards to MACE. In the two primary efficacy analyses, dapagliflozin did not result in a lower rate of MACE $(8.8 \%$ in the dapagliflozin group and $9.4 \%$ in the placebo group; hazard ratio, $0.93 ; 95 \% \mathrm{CI}, 0.84$ to $1.03 ; \mathrm{P}=0.17$ ) but did result in a lower rate of hospitalization for heart failure (hazard ratio, 0.73 ; $95 \% \mathrm{CI}, 0.61$ to 0.88). There was no difference in $\mathrm{CV}$ death between the two groups (hazard ratio, $0.98 ; 95 \% \mathrm{CI}, 0.82$ to 1.17 ). Death related to any cause occurred in $6.2 \%$ and $6.6 \%$ in dapagliflozin and placebo group respectively (hazard ratio, $0.93 ; 95 \% \mathrm{CI}, 0.82$ to 1.04 ). In summary, dapagliflozin when used in T2DM patients who had or were at risk of ASCVD, did not have any clinically meaningful effect on MACE than placebo but did result in a lower rate of hospitalization for heart failure [14]. However, in a sub-group analysis of DECLARE TIMI-58, in patients with prior MI $(n=3,584)$, dapagliflozin reduced the relative risk of MACE by $16 \%$ and the absolute risk by $2.6 \%$ (15.2\% vs. 17.8\%; HR 0.84; 95\%CI 0.72-0.99; p=0.039) [15].

\section{SGLT inhibitors and Heart Failure}

Recently, a meta-analysis was reported looking explicitly at the hospitalization due to heart failure (hHF) in patients using SGLT$2 \mathrm{i}$ looking at randomized controlled trials (RCTs), observational studies, and both. This demonstrated a significant decrease in hHF in RCTs ( $\mathrm{N}=34,322)$, observational studies ( $\mathrm{N}=15,36,339)$, and both $(\mathrm{N}=15,70,661)$ with odds ratio (OR) of $0.70,0.64,0.66$ respectively ( $p=0.000$ for all of them) with SGLT-2i compared to placebo or other anti-diabetes drugs in T2DM [16].

Given the fact that there is multi-model benefit with these drugs in patients with myocardial pump failure, there is growing 
interest currently to study them in patients without diabetes too. "The Dapagliflozin And Prevention of Adverse-outcomes in Heart Failure trial" (DAPA-HF) is one such study which is a prospective, randomized, placebo-controlled trial evaluating the effect of dapagliflozin compared to placebo on morbidity and mortality in patients with heart failure and reduced ejection fraction (HFrEF). Patients with and without T2DM have been included [17]. "The EMPA-TROPISM Trial: Are the 'Cardiac Benefits of Empagliflozin Independent of Its Hypoglycemic Activity?" is a randomized, double-blind, placebo-controlled trial comparing the efficacy and safety of empagliflozin in non-diabetic heart failure patients to determine whether it improves cardiac function and heart failure parameters in these patients [18]

\section{Summary}

Several CV safety trials have been done so far with different classes of antidiabetic agents, but for the first time an oral antidiabetic agent has proven $\mathrm{CV}$ efficacy, which is a major step forward in the armory of oral hypoglycemics. Accordingly, empagliflozin and canagliflozin have now been approved by the US FDA not only for glucose-lowering, but also to reduce the risk of cardiovascular events/mortality in people with T2DM and established CV disease/ high CVD risk. There are several proposed mechanisms behind the CV benefits with these drugs including the obvious ones such as glycemic benefit, weight loss, reduction of blood pressure without tachycardia, diuretic effect with resultant reduction in preload, but also their effects on markers of arterial stiffness, vascular resistance, visceral adiposity, albuminuria, plasma urate and myocardial cell metabolism in patients with heart failure $[19,20]$. Further research is ongoing to potentially expand the usage of these drugs in nondiabetic patients with heart failure as well.

\section{Acknowledgements}

None.

\section{Conflicts of Interest}

No conflict of interest.

\section{References}

1. (2019) https://www.who.int/news-room/fact-sheets/detail/diabetes; accessed.

2. Guifeng Xu, Buyun Liu, Yangbo Sun, Yang Du, Linda G Snetselaar, et al. (2018) Prevalence of diagnosed type 1 and type 2 diabetes among US adults in 2016 and 2017: population based study. BMJ 362: k1497.

3. Aguiree F, Brown A, Cho NH, Dahlquist G, Dodd S, et al. (2013) IDF Diabetes Atlas Pp. 155.

4. (2019) Centers for Disease Control and Prevention. National diabetes statistics report: Estimates of diabetes and its burden in the United States, 2014. Atlanta GA: US Department of Health and Human Services 2014 accessed.
5. Matheus AS, Tannus LR, Cobas RA, Palma CC, Negrato CA, et al. (2013) Impact of diabetes on cardiovascular disease: an update. Int J Hypertens 2013: 653789.

6. Vallon V, Platt KA, Cunard R, Schroth J, Whaley J, et al. (2011) SGLT2 mediates glucose reabsorption in the early proximal tubule. J Am Soc Nephrol 22: 104-112.

7. Merovci A, Mari A, Solis C, Xiong J, Daniele G, et al. (2015) Dapagliflozin lowers plasma glucose concentration and improves $ß$-cell function. J Clin Endocrinol Metab 100: 1927-1932.

8. Singh M, Kumar A (2018) Risks Associated with SGLT2 Inhibitors: An Overview. Curr Drug Saf 13(2): 84-91.

9. Food and Drugs Administration (2008) Guidance for industry diabetes mellitus-evaluating cardiovascular risk in new antidiabetic therapies to treat type 2 diabetes.

10.Zinman B, Wanner C, Lachin JM, Fitchett D, Bluhmki E, et al. (2015) Empagliflozin, cardiovascular outcomes, and mortality in type 2 diabetes. N Engl J Med 373 (22): 2117-2128.

11. Neal B, Perkovic V, Mahaffey KW, Zeeuwet D, Fulcher G, et al. (2017) Canagliflozin and cardiovascular and renal events in type 2 diabetes. $\mathrm{N}$ Engl J Med 377(7): 644-657.

12. Figtree GA, Rådholm K, Barrett TD, Perkovic V, Mahaffey KW, et al. (2019) Effects of Canagliflozin on Heart Failure Outcomes Associated with Preserved and Reduced Ejection Fraction in Type 2 Diabetes: Results from the CANVAS Program. Circulation.

13. Rossignol P, Hernandez AF, Solomon SD, Zannad F (2019) Heart failure drug treatment. Lancet 393(10175): 1034-1044.

14. Wiviott SD, Raz I, Bonaca MP, Mosenzon O, Kato ET, et al. (2018) Dapagliflozin and cardiovascular outcomes in type 2 diabetes. $\mathrm{N}$ Engl J Med 380(4): 347-357

15. Furtado RHM, Bonaca MP, Raz I, Zelniker TA, Mosenzon O, et al. (2019) Dapagliflozin and Cardiovascular Outcomes in Patients with Type 2 Diabetes and Prior Myocardial Infarction: A Sub-analysis From DECLARE TIMI-58 Trial. Circulation.

16. Singh AK, Singh R (2019) Heart failure hospitalization with SGLT2 inhibitors: a systematic review and meta-analysis of randomized controlled and observational studies. Expert Rev Clin Pharmacol 12(4): 299-308.

17. Mc Murray JJV, DeMets DL, Inzucchi SE, Køber L, Kosiborod MN, et al. (2019) A trial to evaluate the effect of the sodium-glucose co-transporter 2 inhibitor dapagliflozin on morbidity and mortality in patients with heart failure and reduced left ventricular ejection fraction (DAPA-HF). Eur J Heart Fail 21(5): 665-675.

18. Santos Gallego CG, Garcia Ropero A, Mancini D, Pinney SP, Contreras JP, et al. (2019) Rationale and Design of the EMPA-TROPISM Trial (ATRU4): Are the "Cardiac Benefits" of Empagliflozin Independent of its Hypoglycemic Activity? Cardiovasc Drugs Ther 33(1): 87-95.

19. Theodosios D Filippatos, Angelos Liontos, Ioanna Papakitsou, Moses S Elisaf (2019) SGLT2 inhibitors and cardioprotection: a matter of debate and multiple hypotheses, Postgraduate Medicine 131(2): 82-88.

20. Garcia Ropero A, Santos Gallego CG, Zafar MU, Badimon JJ (2019) Metabolism of the failing heart and the impact of SGLT2 inhibitors. Expert Opin Drug Metab Toxicol 15(4): 275-285. 